\title{
Review
}

\section{Ribosomal RNA gene repeats, their stability and cellular senescence}

\author{
By Takehiko KoBAYASHI*1,†
}

(Communicated by Shigekazu NAGATA, M.J.A.)

\begin{abstract}
The ribosomal RNA gene (rDNA) repeats form a historically well-researched region in the chromosome. Their highly repetitive structure can be identified easily which has enabled studies on DNA replication, recombination, and transcription. The region is one of the most unstable regions in the genome because of deleterious recombination among the repeats. The ribosomal RNA gene repeats use a unique gene amplification system to restore the copy number after this has been reduced due to recombination. It has been shown that unstable features in the genome can accelerate cellular senescence that restricts the lifespan of a cell. Here, I will introduce a study by our group that shows how the stability of rDNA is maintained and affects lifespan. I propose that the ribosomal RNA gene repeats constitute a center from which the stability of the whole genome is regulated and the lifespan of the cell is controlled.
\end{abstract}

Keywords: ribosomal RNA gene (rDNA), genome stability, cellular senescence, recombination, budding yeast, noncoding transcription

\section{Introduction}

The most abundant genes in cells from bacteria to eukaryotes are those encoding for ribosomal RNA. In bacteria, Escherichia coli, there are 7 ribosomal RNA genes that are spread over the circular genome but they are mainly present in regions near an origin of replication (Fig. 1A). ${ }^{1)}$ The direction of transcription is the same as that of the replication forks to avoid collision. ${ }^{2)}$ In contrast, in eukaryotic cells, the ribosomal RNA genes are organized on the chromosome in huge clusters of tandem repeats, called rDNA (Fig. 1BC). The rDNA produces ribosomal RNA to meet the huge demand for ribosomes that occupy $\sim 50 \%$ of the total protein mass in a cell. ${ }^{3)}$ A ribosome is composed from ribosomal RNA (rRNA) and ribosomal proteins (r-proteins), and translates mRNA to protein. It is a critical machinery for living organisms. The RNA components of a ribosome are working like a ribozyme and play a central role,

*1 National Institute of Genetics, Division of Cytogenetics/ Dept. of Genetics, The Graduate University for Advanced Studies, SOKENDAI, Shizuoka, Japan.

$\dagger$ Correspondence should be addressed: T. Kobayashi, National Institute of Genetics, Division of Cytogenetics/Dept. of Genetics, The Graduate University for Advanced Studies, SOKENDAI, 1111 Yata, Mishima, Shizuoka 411-8540, Japan (e-mail: takobaya@nig.ac.jp). together with aminoacyl tRNA, in polypeptide formation, while the ribosomal proteins maintain the ribosomal structure and regulate the translational process. ${ }^{4)}$ The numbers of each rRNA and r-proteins are almost even in the cell, but in terms of the number of their genes this is very different. For each r-protein there is basically one gene and its mRNA is translated many times while for rRNAs, which will not undergo such a translational amplification process, many genes are required for their mass production. For similar reasons, there are many gene copies for each tRNA, although these do not form a repetitive structure on the chromosome. Due to its repetitive structure, rDNA is easily detected by enzymatic restriction (Fig. 2), as multiple copies of rDNA fragments with a unique length will be generated that can be detected by ethidium bromide staining as a strong band against a fuzzy background of nonrDNA fragments of various sizes (Fig. 2A). Moreover, hybridization with a probe specific for DNA will yield a strong signal which makes it possible to detect the small number of intermediates that are formed during DNA replication and recombination (Fig. 2BC). These features have contributed to unravel molecular mechanisms associated with these processes.5),6)

In this review, I will discuss that rDNA is the most unstable region in the genome and describe how 
A

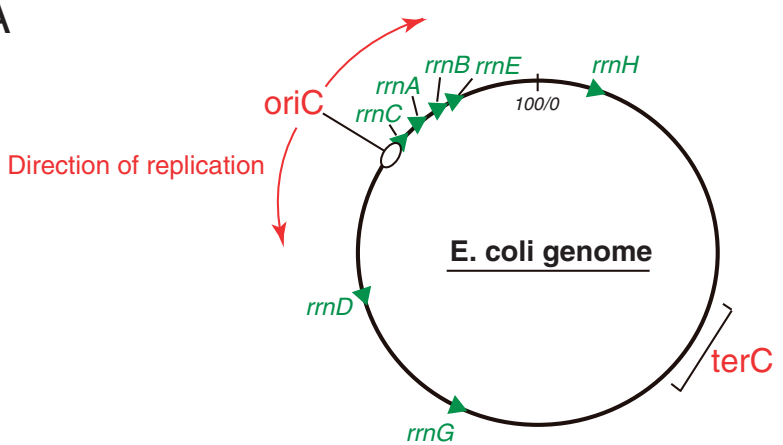

B

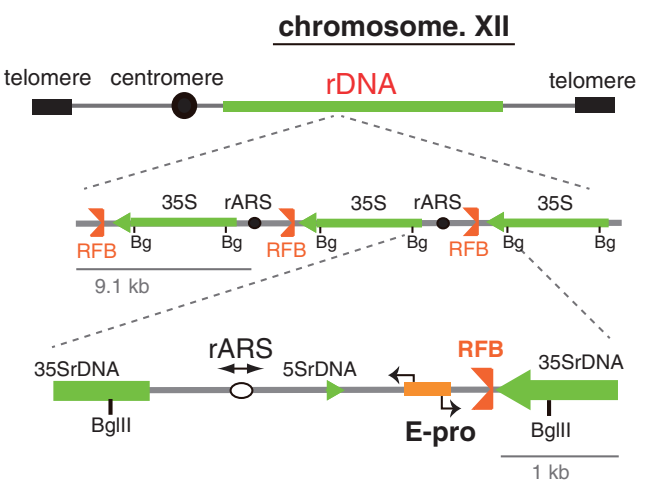

C

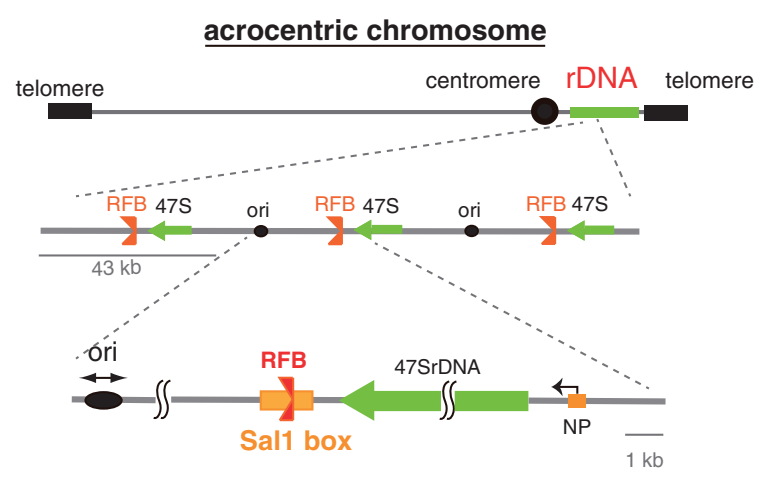

Fig. 1. Structure of rDNA in bacteria, yeast and human cells. (A) In bacteria, Escherichia coli, 7 ribosomal RNA genes are mainly located in a region of the genome that contains an origin of replication. The direction of rRNA transcription (shown by green arrow heads) by RNA polymerase is the same as that of the replication forks (shown by red arrows). (B) In budding yeast (Saccharomyces cerevisiae), an rDNA repeating unit contains a gene for the 35S rRNA precursor that is processed into three mature rRNA species (18S, 5.8S, and 25S rRNA), an rARS (replication origin), a 5S rDNA, an E-pro (non-coding promoter) and an RFB (replication fork blocking site). Restriction by $B g I I \mathrm{I}(\mathrm{Bg})$ produces $\sim 4.6 \mathrm{~kb}$ rDNA fragments. (C) In a human cell, a unit contains an ori (replication origin), an RFB (replication fork blocking site, located in the Sal1 box), the gene for 47S rRNA which is processed into three mature rRNA species (18S, 5.8S, and 28S rRNA) and an NP (non-coding promoter). In yeast, the 5S ribosomal RNA genes localize to the intergenic spacer within the rDNA repeats while in a human cell, these genes are organized in independent repeats.

its stability is maintained. I also discuss how rDNA instability affects other cellular processes, especially cellular senescence.

\section{The structure of rDNA is conserved in eukaryotes}

The ribosomal RNA genes in an eukaryotic cell form clusters with a highly repetitive structure (Fig. 1BC) and are part of the nucleolus in which rRNA is transcribed, processed and assembled with r-proteins into ribosomes. This organization is conserved among most of the eukaryotic organisms. In a human cell, there are five rDNA clusters; on chromosomes 13, 14, 15, 21 and 22 . On these so-called acrocentric chromosomes the centromere is located near one of the telomeres and most of the short arm is occupied by rDNA. The rDNA has a tandem repetitive structure and each cluster has $\sim 70$ repeated units of $\sim 43 \mathrm{~kb}$ each. ${ }^{7), 8)}$ In the well-studied budding yeast (Saccharomyces cerevisiae), often used as the eukaryotic model organism, $\sim 150$ repeats of $\sim 9.1 \mathrm{~kb}$ rDNA units form a single cluster on chromosome XII. $\left.{ }^{9}\right)$ The sequence of the rRNA genes is highly conserved among eukaryotes, while the intergenic spacer regions (IGS) between the genes, although quite different in length and sequence, contain three unique elements that are common; an origin of replication, a replication fork blocking site and a promoter that directs the synthesis of noncoding transcripts. At replication origins, DNA replication starts bi-directionally during $\mathrm{S}$ phase, although only one out of five origins $(\sim 20 \%)$ is actually activated in a cell cycle (For a review see ref. 10) and the active origins are clustered. ${ }^{11)}$ In the 
A

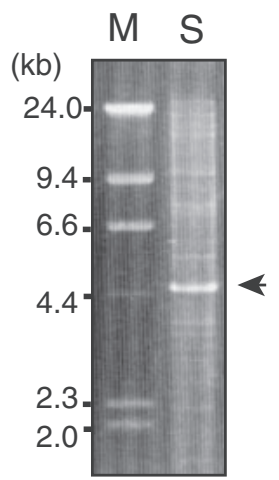

B

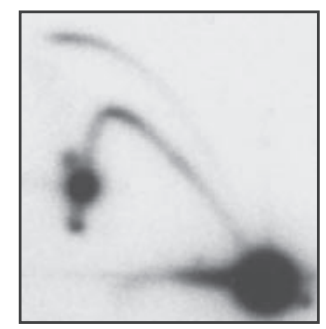

C

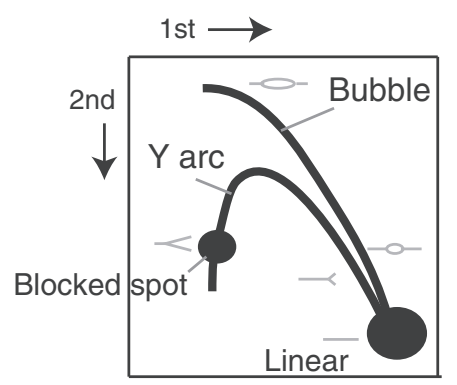

Fig. 2. Detection of rDNA by electrophoresis. Yeast DNA ( $S$. cerevisiae) digested by restriction enzyme $B g l I I$ was analyzed by (A) conventional agarose gel electrophoresis and stained with ethidium bromide or by (B) two dimensional agarose gel electrophoresis that separates replication intermediates. After electrophoresis, southern hybridization was performed to detect the rDNA. (C) A cartoon to explain the pattern of (B). The intermediates (gray) with a branched structure migrate more slowly than linear molecules. In (A), as a size marker, lambda DNA digested by HindIII (M) is run along the digested yeast DNA (S) with the rDNA fragment indicated (arrow head).

case of budding yeast, the origin of replication consists of an $11 \mathrm{bp}$ core sequence that associates with an initiator protein complex (Orc), which opens the double stranded DNA by DNA helicase, after which new strands are synthesized. ${ }^{12}$ The replication fork barrier (RFB) site is quite a unique element in the genome. One of the physiological roles of the $\mathrm{RFB}$ is to avoid collision between replication and transcription machineries. As mentioned below, the transcription of rDNA is quite active, and collision of the RNA polymerase with the progressing replication fork would result in the production of incomplete transcripts. The RFB prevents such a waste of energy and resources. ${ }^{13)}$ The promoter element in the IGS from which the production of non-coding transcripts can be initiated induces gene amplification as discussed below.

\section{Transcription of rDNA and the role of non-transcribed repeat}

The rDNA is the most actively transcribed region in the genome. By electron microscopy using the Miller chromatin spreading technique ("Miller spread"), many intermediates of rDNA transcription can be observed due to their unique appearance (also called "Christmas tree" structures) (Fig. 3). ${ }^{14)}$ In case of budding yeast rDNA, 50 transcription complexes with RNA polymerase I, a specific enzyme for $35 \mathrm{~S}$ rRNA synthesis, are sitting on a single gene. The transcript is processed into three mature ribosomal RNAs (18S, 5.8S, and 25S rRNA), which, together with the $5 \mathrm{~S}$ rRNA that is independently transcribed by RNA polymerase III, establish the backbone of a ribosome. This process is quite active and $\sim 2000$ ribosomes are produced per minute per cell (for review, see ref. 3). There is, however, a long standing mystery about the organization of this process. In spite of the huge demand for transcripts, about half of the rDNA repeats are not transcribed at all (Fig. 3C). That both active and inactive (or silenced) units are present, is common to other organisms. Especially, some plants contain so many rDNA repeats (Table 1 ) that most of them are thought to be inactive. Recently, we identified the reason why so many rDNA units in budding yeast are not active. ${ }^{15)}$ First, we established a series of strains that contained a reduced number of rDNA repeats (20-80 copies) by using a method I will explain later. In these strains, most of the units were transcribed because the demand for rRNA molecules made it such that the cells could not afford to keep inactive rDNA (Fig. 3AB). By careful analysis of possible phenotypes for these "reduced-rDNA" strains, we found that they became sensitive to DNA damaging agents such as methyl methanesulfonate (MMS) and ultraviolet light (UV). The sensitivity to MMS and UV increased as the number of rDNA repeats decreased. The mechanism that explained this observation was quite interesting, that is, the inactive units provide space for repair enzymes to gain access (a "footing place"). In fact, inactive rDNA units were associated with condensin, a protein complex that condenses rDNA and connects sister-chromatids for repair by homologous recombination (Fig. 4). In a strain with 

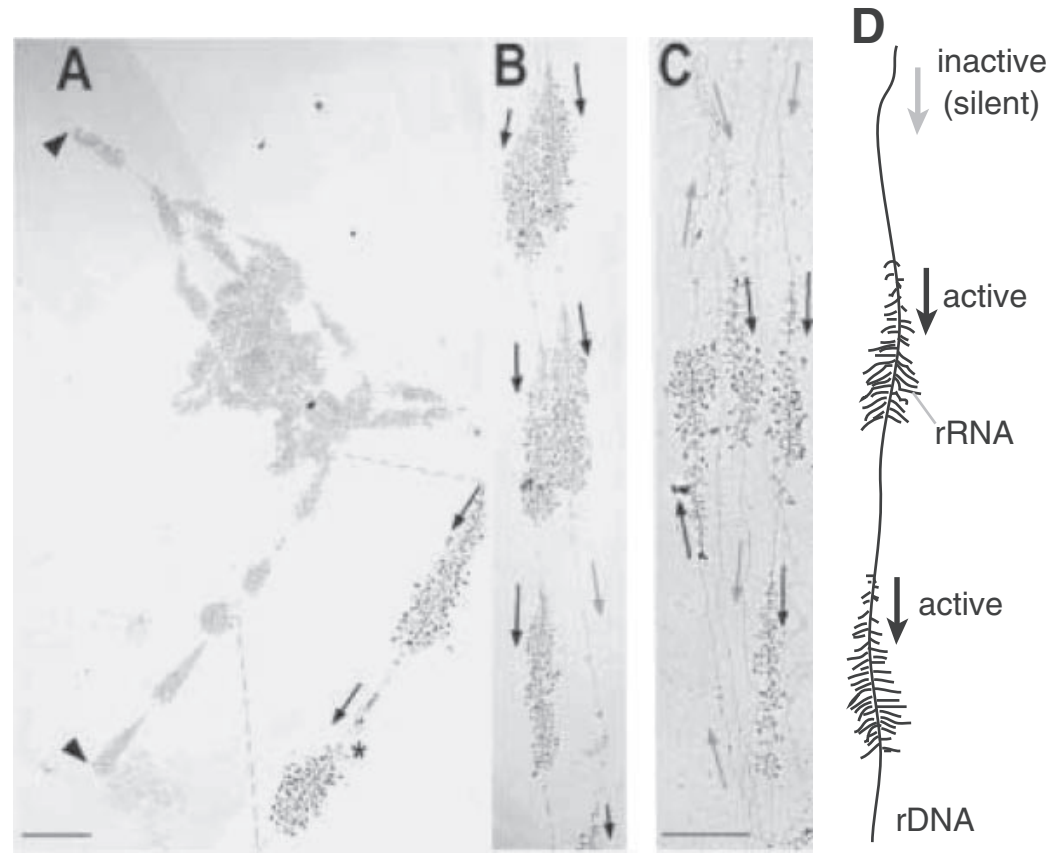

Fig. 3. Transcription of rDNA in budding yeast. Ongoing transcription of rDNA as visualized by electron microscopy (Miller spread) ${ }^{38)}$ in cells from $(\mathrm{A})(\mathrm{B})$ reduced rDNA strains $(\sim 40$ repeats) or $(\mathrm{C})$ the wild type strain $(\sim 150)$. The black and gray arrows indicate the direction of the transcription units in the active and inactive units, respectively. This picture is from French et al. $(2003) .{ }^{14)}$

Table 1. The number of rDNA repeats in several organisms

\begin{tabular}{lc}
\hline \multicolumn{1}{c}{ Species } & $\begin{array}{c}\text { rDNA repeats } \\
\text { no. }\end{array}$ \\
\hline Escherichia coli & 7 \\
Saccharomyces cerevisiae & 150 \\
Drosophila melanogaster & 240 \\
Xenopus laevis & 600 \\
Homo sapiens & 350 \\
Arabidopsis thaliana & 570 \\
Pisum sativum (pea) & 3,900 \\
Zea mays (maize) & 12,000 \\
\hline
\end{tabular}

For review, see Long and Dawid. ${ }^{42}$

a low number of rDNA repeats, which are occupied by the transcription machinery, condensin cannot access the rDNA and any damage that might occur will not be repaired. Thus, as rDNA transcription is so busy, inactive repeats are necessary to allow space for repair machineries.

\section{Copy number maintenance of rDNA}

As the rDNA has a tandem repetitive structure, the repeat number is easily reduced by homologous recombination among the repeats. As shown in Fig. 5A, rDNA damage, such as a double strand break (DSB) caused by radiation, could be repaired by homologous recombination with another unit of a neighboring repeat. During such a crossing-over event, intervening units, which form a loop between the donor and acceptor site, can be excised and the overall number of rDNA repeats will be reduced. Therefore, to maintain the number of rDNA repeats at an optimal level, a cell should have a system to increase (recover) the repeat number. This system is called "gene amplification". In fact, the length of rDNA is always changing in a cyclic manner, as events that lead to the reduction (contraction) of rDNA alternate with those that cause rDNA amplification (expansion) (Fig. 5B). We have studied the molecular mechanisms of rDNA amplification and established the following model. To amplify a gene in the chromosome, a cell needs to replicate some units more than twice and rearrange the extra copies into a repetitive structure. Moreover, these two events have to be coordinated. We found that both the replication initiation and the replication fork blocking activity were critical for this to happen. In the cell cycle, DNA replication starts during $S$ phase from an origin of replication (rARS) bidirectionally. One of the forks (going from left to right in Fig. 6, top) is arrested at the replication fork barrier (RFB) site by the protein Fob1 that 

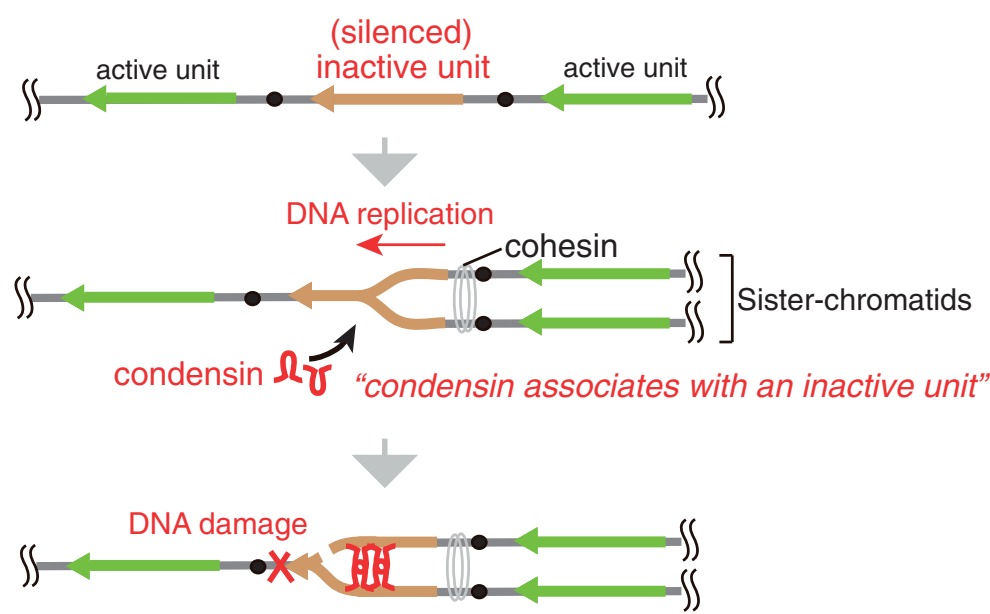

"Replication stalls at DNA damage and DSB is induced"

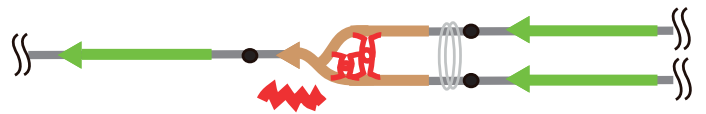

"Condensin helps sister-chromatid recombination for repair"

Fig. 4. Inactive (silenced) units of rDNA provide "working space" for repair. Condensin associates with silenced units and connects sisterchromatids to facilitate recombinational repair. Lack of inactive units reduces the efficiency of repair and increases the sensitivity to DNA damage.

A

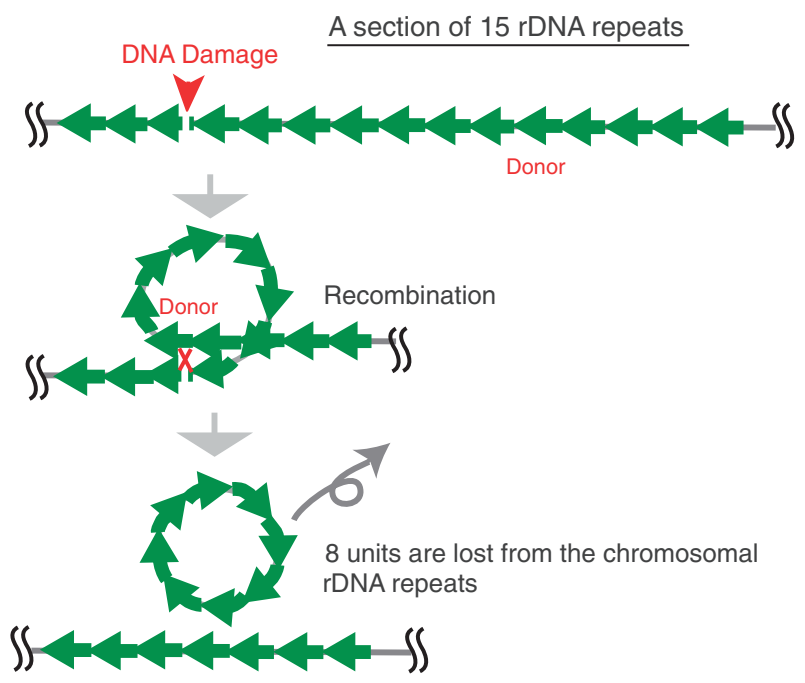

B

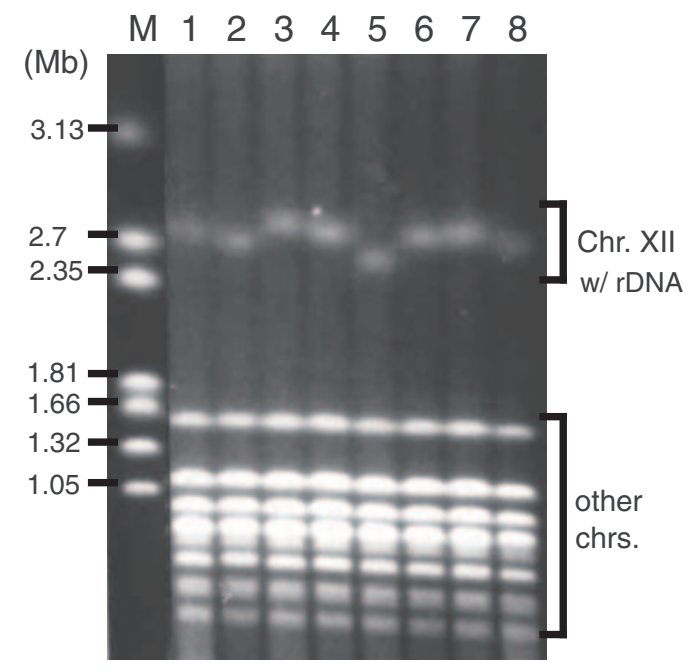

Fig. 5. rDNA repeat number is unstable. (A) DNA damage may reduce the number of rDNA repeats. DNA damage that has occurred in an rDNA unit may be repaired by homologous recombination with another repeat (donor) in the same chromosome. In this case, units between the damage and donor may be lost by crossing-over when DNA is exchanged during recombination. (B) The number of rDNA repeats is always changing. Pulsed-field gel electrophoresis of eight colonies that originated from a single cell. The length of chromosome XII containing the rDNA is different in each colony because of the variation in the number of rDNA repeats. M is a DNA size maker (H. wingei chromosomes). This picture is from Kobayashi (2009). ${ }^{43)}$ 


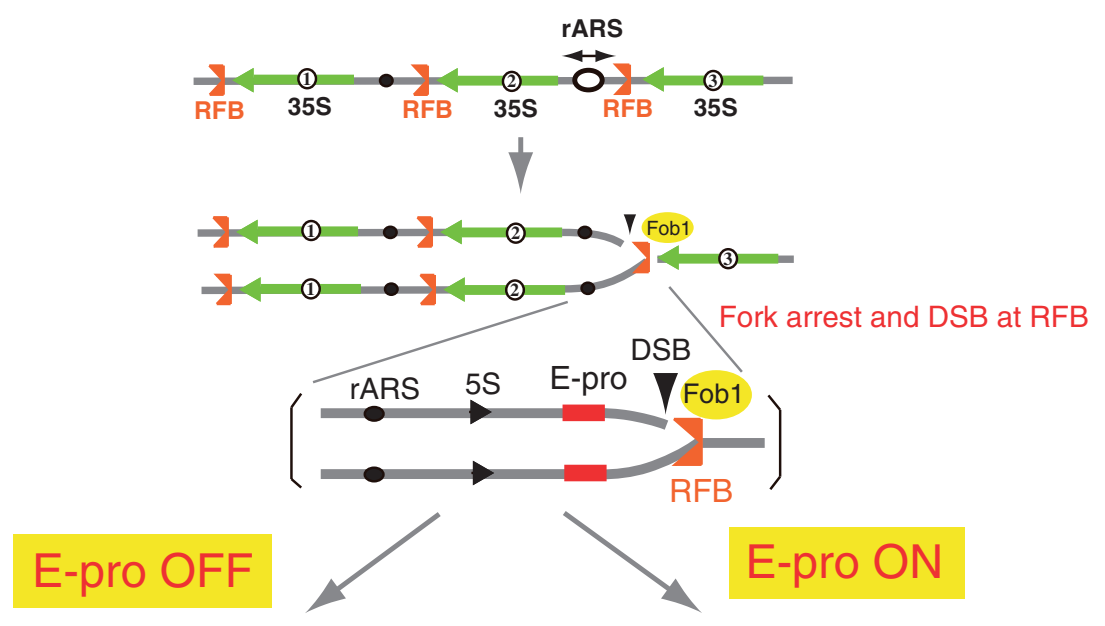

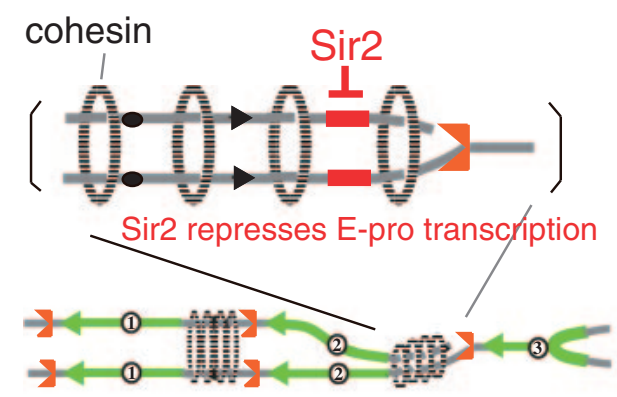

Equal sister-chromatid recombination

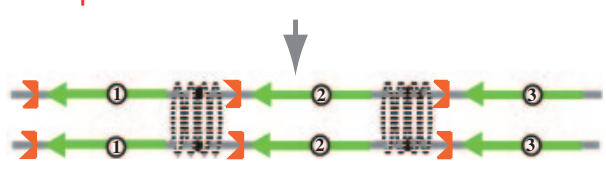

(A) No repeat number changes

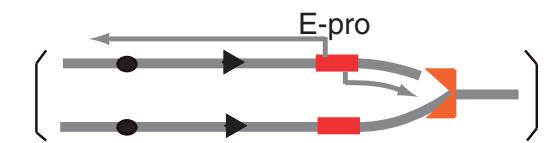

E-pro transcription removes cohesin

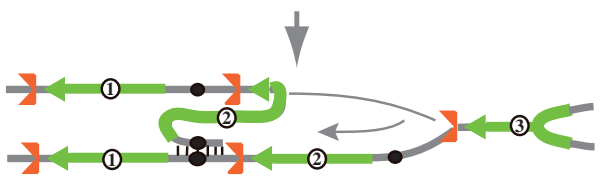

Unequal sister -chromatid recombination

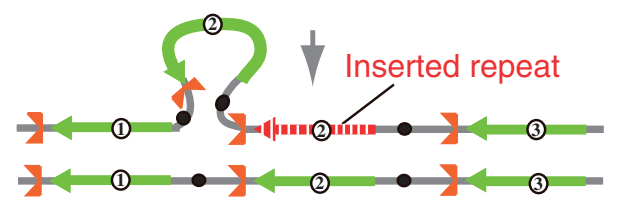

(B) Repeat number increases

Fig. 6. Model of gene amplification in rDNA. (A) In normal situations (rDNA copy number is $\sim 150$ ), the silencing protein, Sir2, represses E-pro (E-pro OFF), allowing the cohesin complex (dotted ellipse) to associate with the IGS. DSBs are repaired by equal sister chromatid recombination, with no change in the copy number. (B) In situations where copy number is reduced, Sir2 repression is removed and E-pro is activated (E-pro ON). The transcription displaces cohesin complex from the IGS and unequal sister chromatids can be used as templates for repair of DSBs. As the result, some units are replicated twice and rDNA copy number increases. The gray lines represent single chromatids (double-strand DNA).

specifically associates with the RFB sequence. Another fork (going from right to left in Fig. 6), however, is not hindered by Fob1 and can pass the RFB. When the fork is arrested, a double strand break (DSB) is induced ${ }^{16), 17)}$ and the broken end is repaired by homologous recombination with the sister-chromatid. In case that the DSB is repaired with the nearest sister-chromatid (this is called equal sister-chromatid recombination), the repeat number does not change. However, in case that the DSB is repaired with a neighboring unit (this is called unequal sister-chromatid recombination), some units are replicated twice and the repeat number increases.
In summary, the RFB coordinates replication and recombination, and through the latter, mediates a possible increase in the number of rDNA repeats.

Recently, we isolated a mutant, rtt109, in which rDNA copy number is highly amplified $(\sim 3$ times greater than the wild type level). ${ }^{18)}$ RTT109 encodes an acetyl transferase that acetylates lysine 56 of histone $\mathrm{H} 3$ and which functions in replicationcoupled nucleosome assembly. In the mutant, we could observe rolling circle-type replication intermediates, thereby suggesting that the coordination of replication and recombination is not working and uncontrollable rolling circle-type replication occurs. 


\section{Regulation of the number of rDNA repeats}

RFB-dependent unequal sister-chromatid recombination will only lead to an increase of the number of rDNA repeats. To keep the number at a proper level, a system that regulates this recombination is also required. The non-coding RNA promoter, E-pro, plays a critical role in this regulation. E-pro was first identified as a sequence which is essential for rDNA amplification as deletion of this sequence completely abolished any increase of the rDNA repeat number. ${ }^{19)}$ Later, we found that transcription from E-pro increased the rate of unequal sisterchromatid recombination by disturbing the association of cohesin, that connects sister-chromatids until cell division, with the rDNA (Fig. 6). ${ }^{16), 20)}$ Interestingly, when the rDNA repeat number reaches the wild-type level, E-pro expression is repressed by Sir2, which is a $\mathrm{NAD}^{+}$-dependent histone deacetylase. In other words, Sir2 regulates the number of rDNA repeats through repression of non-coding E-pro transcription. ${ }^{20)}$

Though it is not known how Sir2 monitors the number of rDNA repeats, I speculate that SIR2 expression itself is regulated by Sir 2 protein. When the number of rDNA repeats reduces, Sir2 is released from the rDNA and binds to the SIR2 promoter to repress transcription. As a result, the level of Sir2 protein in the cell goes down and E-pro can start transcription to activate rDNA amplification. When the repeat number reaches the wild type level, Sir2 can be bound again by the rDNA repeats and is titrated from the SIR2 promoter, leading to an increase in the expression of $S I R 2$ with as end-result the repression of E-pro transcription. ${ }^{21)}$

In a mouse cell, there is a non-coding promoter element in the IGS, too. The transcripts are related to the regulation of $\mathrm{rDNA}$ transcription. ${ }^{22)}$ Though so far there is no evidence that the promoter enhances recombination, it may have the similar role to yeast E-pro because of the highly conserved rDNA structure from yeast to mouse.

\section{rDNA quality is controlled by the rDNA amplification system}

rDNA consists of gene repeats and more than half of these repeats are not transcribed. In general, in repetitive sequences like these, especially in nontranscribed units, mutations can accumulate and turn such units into non-functional pseudogenes. In the case of rDNA, however, all repeats are quite homogeneous, which suggests the presence of a quality control system that prevents the accumulation of rDNA mutations. ${ }^{23)}$ We demonstrated that the variation in the number of $\mathrm{rDNA}$ repeats, discussed above, mediates sequence homogenization. ${ }^{24)}$ We followed the fate of a neutral mutation we introduced at a particular site so that neither rDNA transcription nor its stability would be affected. The mutation either spread over multiple rDNA repeats or was deleted. ${ }^{24)}$ This indicates that the FOB1-induced unequal sister-chromatid recombination controls not only the rDNA repeat number (quantity), but also homogeneity (quality) (for review, see ref. 21).

\section{Fob1 and Sir2 are aging genes}

Fob1 and Sir2 are central players in rDNA maintenance; Fob1 induces recombination to change the number of repeats and Sir2 represses E-pro transcription to keep the number at a proper level. In addition to these roles, Fob1 and Sir2 are known to affect cellular senescence in budding yeast. ${ }^{13), 25), 26)}$ Before continuing the story, I would like to introduce yeast aging. The budding yeast is the simplest organism to show an aging phenotype. A cell (mother) produces a daughter by cell division, called "budding". The daughter cell is smaller than the mother, therefore we can recognize which is the daughter cell after budding (Fig. 7) and follow its fate. After two hours, the daughter cell divides and becomes a mother that produces a daughter. After $\sim 15$ cell divisions, the mother shows aging phenotypes, such as a slow cell division, mitochondrial dysfunction, enlargement of the vacuole (and thereby of its cell-size), and genome instability (for review, see ref. 27 ). After $\sim 20$ cell divisions, in other words, after producing $\sim 20$ daughter cells, the mother cell stops dividing and dies. Going back to the relationship to rDNA, FOB1 and SIR2 affect the rate of aging; a fob1 mutation extends the cellular lifespan by $\sim 60 \%$ while a sir2 mutation shortens the lifespan by $\sim 50 \%{ }^{13), 25), 26)}$ Moreover, overproduction of Sir2 protein extends the lifespan. ${ }^{25)}$ These data suggest that rDNA stability is related to the cellular lifespan.

\section{rDNA stability is the main determinant of yeast lifespan}

To show that a direct relationship exists between rDNA stability and lifespan, we manipulated the stability of the rDNA and monitored what effect this had on lifespan. For this experiment, we replaced the non-coding promoter (E-pro), which is repressed by Sir2, with the galactose promoter 
A

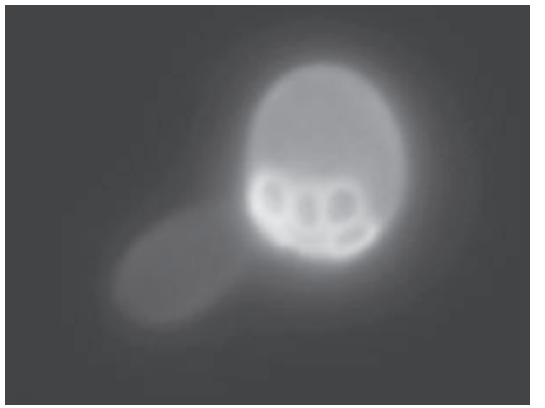

B

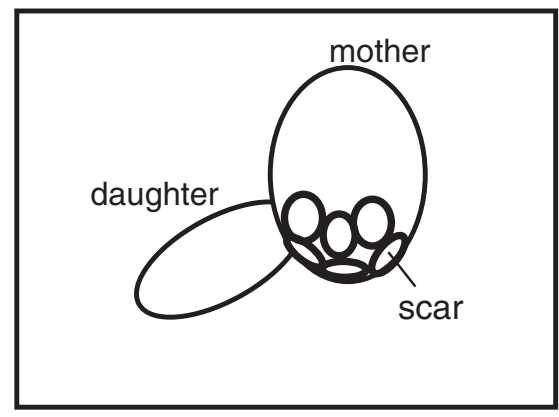

Fig. 7. "Budding" in yeast. (A) A bud on the mother cell grows into a daughter cell. White rings on the surface of the mother cell are scars that are traces of budding. The smaller daughter cell is separated from the mother cell at the end of M phase. (B) A cartoon to explain (A).

(GAL-pro) that is repressed when cells are growing on media with glucose as the carbon source and induced when cells are on galactose medium. Therefore, by choice of carbon source, we can control transcription from the Gal-promoter. The number of repeats in the rDNA is so large that we cannot replace their E-pro with the GAL-pro by means of common gene replacement methods. So, we first reduced the number of rDNA repeats from $\sim 150$ to two, replaced the E-pro with the GAL-pro by gene replacement and amplified the mutant rDNA unit to the wild-type repeat number. As a result, we succeeded in replacing all the E-pro with the GALpro. ${ }^{20)}$ As expected, when cells were grown on galactose as a carbon source and the GAL-pro was activated, the rDNA became unstable whereas the rDNA remained quite stable during growth on glucose when the GAL-pro was silenced. In this setup, SIR2 became dispensable for maintaining rDNA stability. As for the relationship between rDNA stability and lifespan, there was a nice correlation. That is, on galactose, when the rDNA was unstable, lifespan was shortened. In contrast, on glucose, when the rDNA was stable, lifespan was extended. Furthermore, SIR2 was no longer required for a long lifespan. Therefore, we concluded that rDNA stability is a major determinant of lifespan. ${ }^{28)}$

\section{Conserved roles of Sir2 in aging}

As mentioned, rDNA stability affects cellular lifespan. In yeast, Sir2 maintains rDNA stability through its repression of E-pro transcription. Therefore, Sir2 is a primary aging gene. Sir2 homologues have been identified in many organisms. They are $\mathrm{NAD}^{+}$-dependent deacetylases although the targets are not always histones. Recently, it was reported that overexpression of Sir2 homologue SIRT6 extended the lifespan of male mice. ${ }^{29)}$ Despite some disagreement in the literature about the mechanism of how Sir2 homologs act, Sir2 possibly works as a lifespan regulator not only in budding yeast but also in other organisms. ${ }^{30)}$

In budding yeast, SIR2 affects several physiological functions. It was first identified as a silencing gene that down-regulated transcription in telomeres, silent mating type loci, and rDNA. In addition, during cell division, SIR2 induces asymmetrical segregation of proteins so that oxidized, i.e. less functional, proteins stay in the mother cell. ${ }^{31)}$ Although these SIR2-dependent events are also thought to contribute to the processes that determine cellular lifespan, the major determinant in yeast seems to be transcriptional repression of E-pro. This conclusion is, as mentioned above, based on the fact that the lifespan extension obtained after deletion of E-pro did not require the presence of Sir2. In other words, Sir2 maintains lifespan mainly through E-pro repression in the rDNA, not through other events.

\section{The rDNA theory for aging}

What would be the biological meaning of senescence? One possible explanation is that senescence prevents the appearance of abnormal cells. It is known that genome instability (i.e. mutations, rearrangements, etc.) gradually increases by internal (e.g. replication errors) and external (e.g. ultraviolet light) causes through cell divisions. This instability changes expression of genes and possibly induces cancer in mammalian cells. When the lifespan is longer, the risk becomes bigger. Therefore, one physiological role of senescence is to kill cells before such cellular abnormality appears. ${ }^{32)}$ 
DNA damaging factors

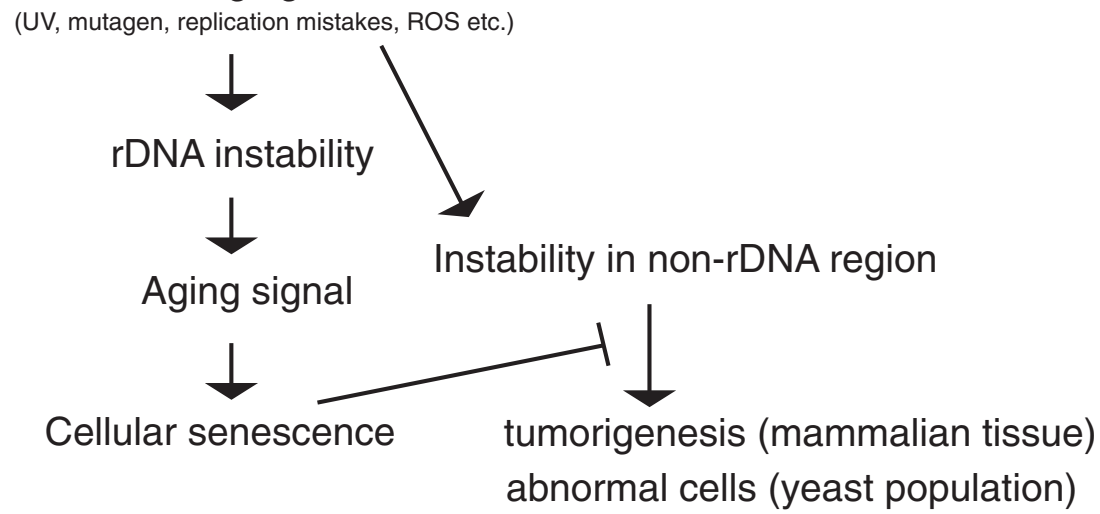

Fig. 8. The rDNA theory for aging. The rDNA region is sensitive to DNA damage by internal and external causes. Through cell divisions, the rDNA becomes more unstable than other parts of the genome and produces an aging signal that tells the cell to die. This precaution prevents the appearance of abnormal cells in a population or a tissue.

I think that the rDNA is the best region to provide indications when to induce cellular senescence. The rDNA is the most unstable region in the genome; it is the most sensitive to genotoxic factors and becomes unstable earlier than other genomic regions. Moreover, it is known that rDNA stability is recovered in a rejuvenated daughter cell. ${ }^{33)}$ Therefore, it is apparently useful for a cell to induce senescence before abnormality is caused by mutations in non-rDNA regions (Fig. 8). I have called this rDNA-driven senescence, the rDNA theory for aging. ${ }^{32)}$ In this theory, rDNA instability dominates the instability of the whole genome and induces senescence to prevent abnormality in the cell.

\section{The rDNA theory in mammalian cells}

The structure of rDNA is highly conserved from yeast to human cells. An amplification machinery (replication blocking activity) was also identified in many organisms. ${ }^{34)}$ Therefore, their rDNA is also thought to be unstable and in need of a quality control system to maintain its integrity and the optimal copy number. In some cancer cells, rearrangement in the rDNA was identified. ${ }^{35)}$ Although there is no direct evidence to correlate rDNA instability with senescence in mammals, in cells from patients with premature aging diseases, rearrangements of rDNA repeats were observed. ${ }^{36)}$ Therefore, in addition to telomere shortening that is observed during senescence in differentiated cells, rDNA instability may be a trigger for cellular senescence. Especially, in stem and germline cells in which telomere shortening is not occurring, rDNA instability is possibly working as an inducer of senescence.

\section{Conclusion}

There are two kinds of highly repetitive regions in the genome; telomeres and rDNA. In some eukaryotic cells, the telomere length is getting shorter by replication and when it becomes less than the threshold, the DNA-damage checkpoint response is activated. This shortening causes arrest of the cell division cycle for repair and senescence is induced. ${ }^{37)-39)}$ Therefore, the region is working as a "counter" to measure the number of cell divisions and to tell the cell when it should die. ${ }^{40)}$ In budding yeast and in human stem and germline cells, telomerase, which contains template RNA to extend the end of the telomeric repeats ${ }^{41)}$ is expressed so that telomere length does not change.

In contrast, the rDNA is working as a "sensor" to monitor genome instability. It is still unknown how the rDNA induces senescense. One possibility is that some aging signals are produced from unstable rDNA. Alternatively, the unstable rDNA may titrate factors that are required for genome maintenance and their shortage is recognized as an aging signal. In any case, more experimental analysis will be required to unravel the precise molecular mechanism of how the aging process of the cell is determined by rDNA instability.

\section{Acknowledgements}

I thank Dr. A. Beyer (University of Virginia) for giving me kind permission to use the picture of electron microscopy (Fig. 3). This work was supported in part by grants-in-aid for Scientific Research (23114002) from the Ministry of Education, Culture, Sports, Science and Technology (MEXT), Japan. 


\section{References}

1) Ellwood, M. and Nomura, M. (1982) Chromosomal locations of the genes for rRNA in Escherichia coli K-12. J. Bacteriol. 149, 458-468.

2) Nomura, M., Morgan, E.A. and Jaskunas, S.R. (1977) Genetics of bacterial ribosomes. Annu. Rev. Genet. 11, 297-347.

3) Warner, J.R. (1999) The economics of ribosome biosynthesis in yeast. Trends Biochem. Sci. 24, 437-440.

4) Hartwell, L.H. (2008) Genetics: From Genes to Genomes, 3rd Edition. McGraw-Hill, U.S.A.

5) Brewer, B.J. and Fangman, W.L. (1988) Mapping replication origins in yeast chromosomes. Bioessays 13, 317-322.

6) Ide, S. and Kobayashi, T. (2010) Analysis of DNA replication in Saccharomyces cerevisiae by twodimensional and pulsed-field gel electrophoresis. Curr. Protoc. Cell Biol. Chapter 22, Unit 22.14.

7) Sakai, M., Ohta, T., Minoshima, S., Kudoh, J., Wang, Y., de Jong, P.J. and Shimizu, N. (1995) Human ribosomal RNA gene cluster: identification of the proximal end containing a novel tandem repeat sequence. Genomics 26, 521-526.

8) Gonzalez, I.L. and Sylvester, J.E. (1995) Complete sequence of 43-kb human ribosomal DNA repeat: Analysis of the intergenic spacer. Genomics 27, 320-328.

9) Kobayashi, T., Heck, D.J., Nomura, M. and Horiuchi, T. (1998) Expansion and contraction of ribosomal DNA repeats in Saccharomyces cerevisiae: requirement of replication fork blocking (Fob1) protein and the role of RNA polymerase I. Genes Dev. 12, 3821-3830.

10) Fangman, W.L. and Brewer, B.J. (1991) Activation of replication origins within yeast chromosomes. Annu. Rev. Cell Biol. 7, 375-402.

11) Pasero, P., Bensimon, A. and Schow, E. (2002) Single-molecule analysis reveals clustering and epigenetic regulation of replication origins at the yeast rDNA locus. Genes Dev. 16, 2479-2484.

12) Araki, H. (2011) Initiation of chromosomal DNA replication in eukaryotic cells; contribution of yeast genetics to the elucidation. Genes Genet. Syst. 86, 141-149.

13) Takeuchi, Y., Horiuchi, T. and Kobayashi, T. (2003) Transcription-dependent recombination and the role of fork collision in yeast rDNA. Genes Dev. 17, 1497-1506.

14) French, S.L., Osheim, Y.N., Cioci, F., Nomura, M. and Beyer, A.L. (2003) In exponentially growing Saccharomyces cerevisiae cells, rRNA synthesis is determined by the summed RNA polymerase I loading rate rather than by the number of active genes. Mol. Cell. Biol. 23, 1558-1568.

15) Ide, S., Miyazaki, T., Maki, H. and Kobayashi, T. (2010) Abundance of ribosomal RNA gene copies maintains genome integrity. Science 327, 693-696.

16) Kobayashi, T., Horiuchi, T., Tongaonkar, P., Vu, L. and Nomura, M. (2004) SIR2 regulates recombi- nation between different rDNA repeats, but not recombination within individual rRNA genes in yeast. Cell 117, 441-453.

17) Burkhalter, M.D. and Sogo, J.M. (2004) rDNA enhancer affects replication initiation and mitotic recombination: Fob1 mediates nucleolytic processing independently of replication. Mol. Cell 15, 409-421.

18) Ide, S., Saka, K. and Kobayashi, T. (2013) Rtt109 prevents hyper-amplification of ribosomal RNA genes through histone modification in budding yeast. PLoS Genet. 9, e1003410.

19) Kobayashi, T., Nomura, M. and Horiuch, T. (2001) Identification of DNA cis-elements essential for expansion of ribosomal DNA repeats in Saccharomyces cerevisiae. Mol. Cell. Biol. 21, 136-147.

20) Kobayashi, T. and Ganley, A.R. (2005) Recombination regulation by transcription-induced cohesin dissociation in rDNA repeats. Science 309, 15811584 .

21) Kobayashi, T. (2011) Regulation of ribosomal RNA gene copy number and its role in modulating genome integrity and evolutionary adaptability in yeast. Cell. Mol. Life Sci. 64, 1395-1403.

22) Mayer, C., Schmitz, K., Grummt, I. and Santoro, R. (2006) Intergenic transcripts regulate the epigenetic state of rRNA genes. Mol. Cell 22, 351-361.

23) Ganley, A.R. and Kobayashi, T. (2007) Highly efficient concerted evolution in the ribosomal DNA repeats: total rDNA repeat variation revealed by whole-genome shotgun sequence data. Genome Res. 17, 184-191.

24) Ganley, A.R. and Kobayashi, T. (2011) Monitoring the rate and dynamics of concerted evolution in the ribosomal DNA repeats of Saccharomyces cerevisiae using experimental evolution. Mol. Biol. Evol. 28, 2883-2891.

25) Kaeberlein, M., McVey, M. and Guarente, L. (1999) The SIR2/3/4 complex and SIR2 alone promote longevity in Saccharomyces cerevisiae by two different mechanisms. Genes Dev. 13, 2570-2580.

26) Defossez, P.A., Prusty, R., Kaeberlein, M., Lin, S.J., Ferrigno, P., Silver, P.A., Keil, R.L. and Guarente, L. (1999) Elimination of replication fork block protein Fob1 extends the life span of yeast mother cells. Mol. Cell 3, 447-455.

27) Jazwinsky, S.M. (2001) New clues to old yeast. Mech. Ageing Dev. 122, 865-882.

28) Saka, K., Ide, S., Ganley, A.R. and Kobayashi, T. (2013) Cellular senescence in yeast is regulated by rDNA noncoding transcription. Curr. Biol. 23, $1794-1798$.

29) Kanfi, Y., Naiman, S., Amir, G., Peshti, V., Zinman, G., Nahum, L., Bar-Joseph, Z. and Cohen, H.Y. (2012) The sirtuin SIRT6 regulates lifespan in male mice. Nature 483, 218-221.

30) Lombard, D.B., Pletcher, S.D., Canto, C. and Auwerx, J. (2011) Longevity hits a roadblock. Nature 477, 410-411.

31) Aguilaniu, H., Gustafsson, L., Rigoulet, M. and Nyström, T. (2003) Asymmetric inheritance of oxidatively damaged proteins during cytokinesis. 
Science 299, 1751-1753.

32) Kobayashi, T. (2008) A new role of the rDNA and nucleolus in the nucleus-rDNA instability maintains genome integrity. Bioessays 30, 267-272.

33) Ganley, A.R.D., Ide, S., Saka, K. and Kobayashi, T. (2009) The effect of replication initiation on gene amplification in the rDNA and its relationship to aging. Mol. Cell 35, 683-693.

34) Dalgaard, J.Z., Godfrey, E.L. and MacFarlane, R.J. (2011) DNA Replication-Current Advances. Edited by Herve Seligmann, InTech, Chapter 13.

35) Stults, D.M., Killen, M.W., Williamson, E.P., Hourigan, J.S., Vargas, H.D., Arnold, S.M., Moscow, J.A. and Pierce, A.J. (2009) Human rRNA gene clusters are recombinational hotspots in cancer. Cancer Res. 69, 9096-9104.

36) Caburet, S., Conti, C., Schurra, C., Lebofsky, R., Edelstein, S.J. and Bensimon, A. (2005) Human ribosomal RNA gene arrays display a broad range of palindromic structures. Genome Res. 15, 10791085.

37) Takai, H., Smogorzewska, A. and de Lange, T. (2003) DNA damage foci at dysfunctional telomeres. Curr. Biol. 13, 1549-1556.

38) D'adda di Fagagna, F., Reaper, P.M., Clay-Farrace,
L., Fiegler, H., Carr, P., von Zglinicki, T., Saretzki, G., Carter, N.P. and Jackson, S.P. (2003) A DNA damage checkpoint response in telomere-initiated senescence. Nature 426, 194-198.

39) Herbig, U., Jobling, W.A., Chen, B.P., Chen, D.J. and Sedivy, J.M. (2004) Telomere shortening triggers senescence of human cells through a pathway involving ATM, p53, and p21(CIP1), but not p16(INK4a). Mol. Cell 14, 501-513.

40) Kobayashi, T. (2011) How does genome instability affect lifespan?: roles of rDNA and telomeres. Genes Cells 16, 617-624.

41) Greider, C.W. and Blackburn, E.H. (1985) Identification of a specific telomere terminal transferase activity in Tetrahymena extracts. Cell 43, 405413.

42) Long, E.O. and Dawid, I.B. (1980) Repeated genes in eukaryotes. Annu. Rev. Biochem. 49, 727-764.

43) Kobayashi, T. (2009) Collaboration of replication, recombination and noncoding transcription for gene amplification. Protein, Nucleic Acid Enzyme $\mathbf{5 4}, 537-542$.

(Received Nov. 22, 2013; accepted Jan. 8, 2014)

\section{Profile}

Professor Takehiko Kobayashi was born in Yokohama, Japan in 1963. He received a B.Sc. degree in biology from Kyushu University in 1987. In 1992, he obtained a Ph.D. from the same university under the supervision of Prof. T. Horiuchi. After working three years as a postdoc with Dr. Melvine DePamphilis at Roche Institute of Molecular Biology in New Jersey and National Institute of Health in Maryland, USA, he returned to Japan as an assistant professor in the research group of Prof. T. Horiuchi in 1997. He was promoted to associate professor in 2004. In 2006, he moved to National Institute of Genetics in Mishima, Japan as a full professor. His research interest is the relationship between genome stability, cellular senescence and rejuvenation. He has received awards, "Prize for Science and Technology" by the Ministry of Education, Culture, Sports,

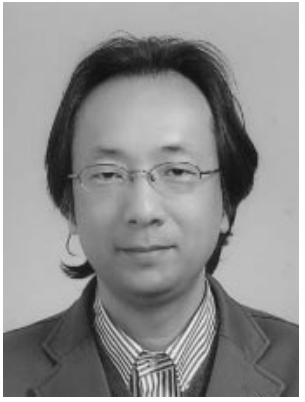
Science and Technology, Japan in 2012 and "the 29th Inoue prize" by Inoue Foundation for Science in 2013. 